\title{
Analysis of Vehicle Collision Safety and Its Security System Yu Qian
}

School of Automotive Engineering, Wuhan University of Technology, Wuhan, Hubei, 430000

\author{
Keywords: Vehicle Collision Safety, Security Data Collection, Analysis
}

\begin{abstract}
The vehicle crash test is the main means of vehicle safety research. Any passive safety research is based on the data measured by the trolley or real vehicle crash test. The electrical measurement of the vehicle crash test instrument is mainly a data collector. Car crash test using electrical measuring instruments and ordinary instruments are many different. It must be able to meet the displacement, speed, high acceleration and so on. In recent years, the world's in-vehicle instrumentation company has improved the existing test system, including multi-channel signal conditioning, analog-digital conversion, storage function, through the application of the latest technology, the test system size and weight reduction.
\end{abstract}

\section{Introduction}

Urban traffic on any road environment are likely to occur traffic accidents and show a certain characteristics and laws. Urban traffic accidents are frequent for the evening to early morning, accident-prone areas for the suburbs. Urban roads generally include facilities of various types, various grades of roads and other facilities, in the highly developed modern city traffic, urban roads also include elevated roads, pedestrian crossings (tunnels) and large interchange facilities and other facilities. Traffic accidents in urban roads are mainly intersections and basic sections. The main intersections are rounds, intersections, tibs intersections, and so on. For the main types of intersections, there are frontal collisions and side collisions. The main collision type is a frontal collision.

\section{Analysis of Collision Predictive Technology}

Compared with the traditional passive safety system, the significant advantage of the application of the collision prediction is reflected in two aspects: (1) Since it is possible to find the potential of the collision in advance of the accident, the reversible passive safety protection device (2) the collision information obtained by the radar in advance can be controlled by the traditional passive safety protection device, etc. (2) the collision information obtained by the radar in advance can be used to control the traditional passive safety protection device Has brought more positive effects, not only to determine the environment before the accident more accurate, but also make these devices trigger a better time to choose, such as airbag point burst algorithm can be a great degree of optimization.

Mercedes-Benz has used the same model for Pre-safe validity of the comparative test. The head of the test crew is used as a comparison standard. Test results show that the installation of Pre-safe, the crew's head displacement reduced to the original 1/4 below the level, greatly enhance the occupant's protective effect. Since 2003, foreign major automobile companies such as Toyota, Mercedes-Benz, etc. have been launched their own application of the concept of collision in the high-end models, and put into the market. Is expected in the next 10 years, will become active security and passive security field of a key technical direction, and gradually to the low-end models to promote, but the current domestic research in this area is still in its infancy.

The entire system consists of three modules: radar detection module, body bus network and the end of the passive safety device. Among them, the body CAN bus network is already very mature and widely used technology, so the main focus of the current research focused on the front of the radar system and the rear of a variety of end of the implementation of the device above.

Through the forward radar (laser radar or millimeter-wave radar, where the millimeter-wave radar in the future application will tend to become mainstream) to measure the information in front 
of the vehicle, and this information through the CAN network to pass such as ECU master unit, the ECU to run radar information Screening algorithm and collision prediction algorithm to calculate the prediction of the possibility of collision results, and the results broadcast to the end of the many passive safety devices such as reversible seat belt preload device, airbag, brake, active seat , Early warning, etc., in order to achieve the full protection of the occupants.

\section{Collision Accident Analysis System Development}

The system is developed in Windows XP environment using Visual C ++6.0 programming language, and finally the application software that can realize vehicle collision accident simulation analysis under urban road environment condition. Because Visual C ++6.0 is a set of completely independent of the development system of advanced programming language, it has a visualization, the use of object-oriented programming methods, and operating system compatibility is better, can easily form a variety of users to design software Interface and other advantages. The main functions of the urban road vehicle crash accident analysis system should be realized: 1) The basic parameters of the accident vehicle input, including the type of vehicle (passenger car, passenger car, truck), vehicle quality, vehicle length, vehicle width, Distance, braking type (all wheel brake, front wheel brake, rear wheel brake), road adhesion coefficient, rolling resistance coefficient, vehicle plastic deformation and other data; 2) urban road environmental parameters input part, (Such as cement, gravel, asphalt, dirt road, ice and snow, etc.), road type (straight, corners, slope, etc.), weather conditions (sunny, cloudy, rain, snow, etc.), road conditions 3) According to the mathematical model of the accident before the collision of vehicle speed analysis and analysis; 4) According to the speed of the calculation, the intersection of the intersection, the intersection of the intersection, the intersection, etc.), with or without guardrail and green belt and other scene parameters; Results and environmental parameters, through the scene design, to achieve accident simulation analysis.

According to the function structure of the accident system, the functional modules of the system are divided into three modules: vehicle collision accident parameter input, velocity calculation and accident simulation analysis. The speed calculation module can be divided into positive collision according to the different forms of urban road collision, rear-end collision and side impact of three parts. The velocity calculation analysis is based on the vehicle collision mechanics model. The frontal collision and the rear collision are estimated by the distance of the collision after the collision of the vehicle. Then, the effective collision speed is calculated by the parameters collected in the field, And finally on the basis of the momentum theorem to solve the vehicle speed before the collision; body in the side of the collision, along the normal and tangential direction of the contact with each other to produce force and reaction force, resulting in momentum transmission, the same according to the momentum theorem The normal and tangential equations are established to obtain the state parameters before the collision.

\section{Test System for Vehicle Collision Test}

The research of automobile collision began to meet the requirements of the frontal collision regulation of the automobile, and gradually extended to the side impact and the member protection. In addition, the conditions of the outdoor scientific research experiment were taken into account, which put forward higher requirements for the adaptability of the vehicle test system, Such as impact. Ambient temperature recording time, data protection, data exchange platform and battery characteristics.

Collision test is the final way to test the performance of the collector and the collision test can fully test the collection of the collector, trigger and impact resistance and other functional characteristics. The collector for the car 16-channel collector has been carried out several crash tests, the collision speed from $20 \mathrm{~km} / \mathrm{h}$ to $50 \mathrm{~km} / \mathrm{h}$, seized the usual test of the collision speed range. The test using a trolley, at some typical test points, while the installation of these two sets of vehicle-mounted acquisition system for testing, in order to get the test contrast. Frontal collision crew protection test data include: body and dummy electric measurement data, ie, ECU, engine, 
steering wheel axial, dashboard, firewall floor and brake calipers acceleration, dummy head, chest and pelvic acceleration, stern compression Volume and thigh force and other parameters. The trial used two FTSS50 percentile Hybridl dummies.

\section{Vehicle Crash Safety Collaborative Simulation Analysis}

In the investigation and visit of the automobile enterprises, it was found that during the design and development of the automobile, the parts and components were designed according to the requirements of the whole vehicle, and the geometric model of the parts was submitted to the whole vehicle. The vehicle geometric model was assembled by the whole vehicle, the finite element model is established and the collision simulation test is carried out. According to the test results, the supplier is required to correct the part model and then the collision simulation test is carried out until the collision result reaches the design requirement. In this development process, the parts can not touch the vehicle (Such as parts to modify the design technology, etc.), the supplier's intellectual property rights are not effectively protected. According to the characteristics and needs of the automobile industry, this paper puts forward the data of the automobile industry, As shown in Figure 1, the application prototype is composed of a VO (virtual organization) containing vehicle operators, component providers, resource providers (providing computing resources and temporary file storage resources). The entire collision collaborative simulation process is a loosely coupled Asynchronous dynamic collaborative process. Specific steps are as follows:

(FA) model (finite element model), component environment CAD model and FE model, which are used to establish the input model of the vehicle to be designed, in which the environmental model refers to a model that is connected or may be in contact with the part to be designed. Component parts. Part parts design components. Parts suppliers to obtain vehicle parts to provide the parts of the environment model and FE model, the use of these models in the local platform design and component environment model coordination of the parts model, the establishment of parts FE model, and with the resulting component environment FE model is assembled to ensure that the component FE model can be loaded into the vehicle model.

C. Collision simulation test. Vehicle remote assembly vehicle FE model and component FE model, in the resource at the collision simulation calculation, vehicle manufacturers and parts manufacturers can remotely monitor the simulation process in real time.

D. Collision simulation results processing. After the collision simulation calculation is completed, the parts results and the vehicle results are decomposed from the overall calculation results. The parts supplier can only obtain the part result, check whether the result of the parts is qualified, and if the vehicle is qualified, (Including the final design results of the parts manufacturer), the simulation process is completed; otherwise the process repeats steps $\mathrm{b} \sim \mathrm{d}$. In the collision safety collaborative simulation workflow, in order to protect the vehicle and parts business intellectual property rights, Assembly and simulation results are decomposed, and the vehicle operator and component dealer shall follow the following conventions: a. The vehicle operator specifies the ID range of the component finite element model for the component, for the node, unit, part, material, connection, contact Etc. The component environment model contains only information about the connection to the component, and the parts supplier can not obtain more vehicle data from it to protect the vehicle's intellectual property rights.

E. The vehicle operator can only obtain the final part results and can not get the middle of the process of data related parts to protect the intellectual property rights of parts manufacturers.

\section{Conclusion}

With the rapid development of China's auto industry, China's civilian car ownership also increased significantly, at the same time, the country's annual traffic accidents are also increasing, and even a number of vicious traffic accidents caused the people's attention. However, under the current national conditions in China, the fundamental causes of the frequent occurrence of automobile accidents in the foreseeable future will inevitably exist for a long time, and therefore, for our 
country, the road traffic accident has actually harmed the national security, car safety Sexual research to improve the safety of passengers and pedestrians pedestrian safety indicators is extremely necessary and extremely urgent. If you can integrate the main and passive safety of the two aspects of the research results in the collision pre-radar system research and development, new passive safety device application or optimization and system integration design of the three key technical aspects of investment, it will be able to Make a difference. It is foreseeable that the vehicle collision prediction system has broad application prospects.

\section{References}

[1] Xi Haifeng, Chen Hua, Jian Jie. Study on Management Pattern of Automobile Production Process Based on RFID Technology [J]. Combined Machine Tool and Automated Processing Technology, 2013 (11)

[2] Ming Xian Cheng, Mei Xiaojun, Yang Jindong. "Ming Minghuan" principle in equipment prevention and maintenance management application [J]. Electrical and Mechanical Engineering Technology, 2013 (07)

[3] Fan Mengzong, Zhang Xianyang, Zhu Feixiong. Study on the process management scheme of automobile performance research and development [J]. Technology and Enterprise, 2013 (13)

[4] Application of R \& D Process Management Technology in Powertrain Development [J]. Design and Manufacture of Diesel Engine, 2013 (02)

[5] Chen Qian. Process management in the laboratory quality management application [J]. Technology to get rich guide, 2013 (17)

[6] Su Hua. The system process management of customer complaints processing [J]. Management modernization, 2012 (04) 